\title{
Soluble Carbohydrates Repress the Cellulolytic Activity of Clostridium cellulovorans and Eubacterium
} cellulosolvens

\author{
Benjamin Gay Blair ${ }^{1}$ and Kevin Lee Anderson ${ }^{2}$ \\ 1. Department of Biology, Jacksonville State University, Jacksonville AL 36265, USA \\ 2. Department of Biology, Arkansas State University, Beebe AR 7201, USA
}

\begin{abstract}
Studies have provided indirect evidence that cellulolytic activity of some anaerobic bacteria is repressed by carbohydrates, such as glucose. This effect is known as carbon catabolite repression (CCR). Previous work has found that cellulolytic activity of Clostridium cellulovorans and Eubacterium cellulosolvens are regulated. Many cellulolytic systems of these organisms are expressed only in the presence of cellulose or cellobiose (the disaccharide of cellulose). Some of these cellulose-induced systems also appear subject to CCR when more soluble substrates, such as glucose, are also available. To determine if such repression directly effects cellulolytic activity of $C$. cellulovorans and E. cellulosolvens, these organisms were cultivated in media containing a glucose analog. We then measured the ability of low levels of analog to inhibit growth of the organisms when cellobiose or cellulose were the energy substrates. Our results found that growth of both C. cellulovorans and E. cellulosolvens in cellobiose-containing medium are strongly inhibited by glucose analogs. In addition, both organisms exhibited delayed and slower growth in cellulose-containing medium when a glucose analog was added. These results provide direct demonstration that these cellulolytic bacteria are subject to CCR. This repression of cellulolysis may affect both of these organisms' ability to serve as industrial platforms for biomass degradation, and may interfere with the contribution of E. cellulosolvens toward animal digestion of cellulose. These results were also in sharp contrast to what has been reported regarding CCR activity in Clostridium cellulolyticum, which actively expresses cellulases in the presence of low levels of glucose.
\end{abstract}

Key words: Cellulolytic, anaerobic bacteria, catabolite repression, cellulolysis, cellulose degradation.

\section{Introduction}

Plant polysaccharides are the earth's most abundant form of renewable biomass. Cellulolytic microorganisms have a significant role in this biomass renewal and the global carbon cycle. Many biofuel platforms, livestock production strategies and recycling protocols also rely upon the successful performance of these microorganisms. Yet, consistent and high yield cellulolytic activity has frequently proven a challenge in these areas.

Microbes regulate their cellulolytic activity to more efficiently utilize all available energy sources within

Corresponding author: Kevin Lee Anderson, Ph.D., assistant professor, research field: microbiology. E-mail: klanderson@asub.edu. the biomass, especially when such resources are limited. A significant group of these cellulolytic organisms are anaerobic bacteria, which are found in a wide variety of ecological niches. Thus, it is important to understand how these bacteria regulate their hydrolytic activity and obtain optimal cellulolysis (i.e., growth using cellulose as the substrate).

Cellulolytic bacteria employ a variety of regulatory systems that can affect their cellulolytic activity and thereby alter their rate of cellulose degradation. This would subsequently alter their overall contribution to biomass recycling and plant digestion. Understanding how these regulatory events can alter bacterial cellulolysis is necessary for a greater understanding of technological, ecological and agricultural advances of biomass conversion. 
A common regulatory system found in many bacteria is the specific selection of substrates. Originally known as the "glucose effect" [1], this mechanism enables some bacteria to preferentially catabolize certain energy substrates, e.g., Escherichia coli has preference for glucose even when lactose is readily available $[2,3]$. This "glucose effect" is often referred to as carbon catabolite repression (CCR) [4, 5]. Depending upon the bacterial species, CCR involves one or a combination of mechanisms in gram-positive bacteria [2-8]. The repression can also be activated by catabolites other than glucose $[2,8,9]$.

The CCR system imposes a hierarchy on the utilization of energy resources. Highly soluble catabolites, such as glucose, typically have the higher preference. Simultaneously, utilization of less preferred catabolites is partly or completely inhibited. This allows a greater level of the cell's metabolic resources to be directed toward the more desired substrate. In addition, for at least some bacteria, CCR provides a balancing system to maintain the catabolite influx within the cell's metabolic capacity range [10].

Several studies have attempted to demonstrate whether a CCR system helps regulate the cellulolytic activity of various anaerobic bacteria. In some of these studies, loss of some cellulose-induced systems was detected when soluble carbohydrates were present in the medium [11-13]. While this suggests a concurrent loss of cellulolysis, it does not provide direct demonstration. In other studies, reduction of specific gene transcripts or reduction in the formation of cellulosome components were detected if glucose was present, but actual reduction of cellulolysis was not tested [12, 14-16]. Furthermore, the results of many such studies are confounded by changes in the medium $\mathrm{pH}$, nutrient competition and the lack of sensitive methods for measuring substrate depletion [17].

Clostridium cellulolyticum has become a standard model for studying cellulolytic clostridia. However, a detailed analysis of C. cellulolyticum found it regulates cellulolytic activity different than what had been reported for other cellulolytic clostridia and even previously reported for C. cellulolyticum [18]. When cultivated in low concentrations of glucose, $C$. cellulolyticum actually expresses high levels of many cellulases, and CCR appears inhibited [18]. While the majority of cellulosomal genes in C. cellulolyticum appear to be regulated by a form of CCR, the relationship of CCR to a reduction of cellulolysis could only be inferred. Thus, the researchers acknowledged that the impact of CCR on the cellulolytic activity of $C$. cellulolyticum is "unclear" [18].

Our study sought to determine what effect (if any) CCR might have on cellulolysis of some anaerobic bacteria. Clostridia comprise a large proportion of the cellulolytic bacteria that have been studied [19], and they potentially provide a significant contribution to biomass recycling in anaerobic environments. Thus, our study included Clostridium cellulovorans, which has served as a model system for the study of mesophilic cellulolytic clostridia [15]. We also studied Eubacterium cellulosolvens, which has been a valuable model system for non-clostridial cellulolytic bacteria $[12,20,21]$, and its inclusion provided a phenotypic range for comparison.

Previous studies have shown that both $C$. cellulovorans and E. cellulosolvens form an exocellular protuberance (possibly a polycellulosome) when cellulose serves as the energy substrate $[12,13$, 22]. However, these protuberances could not be microscopically detected in either cellobiose-grown or glucose-grown cultures, and were rapidly lost (within approximately $5 \mathrm{~min}$ ) if glucose or a glucose analog was added to a cellulose-grown culture $[12,13]$. In addition, other cellulose-inducible components, such as specific lectin binding sites and a cellulose-binding protein fraction, were also rapidly lost when either glucose or a glucose analog was added to a cellulose-grown culture $[12,13]$. The addition of various mono and disaccharides to cellulose-grown cultures of C. cellulovorans also gave a significant 
reduction of some cellulase transcripts [22].

These results would suggest that soluble carbohydrates, such as glucose, can inhibit the cellulolytic activity of these two organisms. Do $E$. cellulosolvens and C. cellulovorans possess a form of CCR that will reduce their overall cellulolysis? To answer this question, we used an approach that employed analogs of glucose.

The identical measured physiological responses of both $C$. cellulovorans and E. cellulosolvens to the presence of glucose or glucose analogs [12, 13] suggest that these bacteria respond to both of these molecules in a similar manner. Thus, glucose analogs could serve as catabolic imitators of glucose. As an imitator, analogs can be used to experimentally assess the activity of CCR in these bacteria without invoking various confounding factors that often result from glucose catabolism (e.g., reduction in medium $\mathrm{pH}$, which is inhibitory of cellulose degradation) [17]. Glucose analogs have been extensively used to detect regulatory events in yeast [23] and various non-cellulolytic bacteria [24, 25]. However, they have not routinely been used to study regulatory activity of cellulolytic bacteria. In our study, the use of glucose analogs provided a means of directly measuring what effect CCR may impose on bacterial cellulolysis.

\section{Materials and Methods}

\subsection{Bacterial Strains and Cultivation}

E. cellulosolvens 5494 (obtained from Mark Rasmussen, ARS/USDA, Ames, IA) and $C$. cellulovorans 743B (ATCC 35296) are both gram-positive, anaerobic, cellulolytic bacteria [26, 27]. Each organism was anaerobically cultivated at $39{ }^{\circ} \mathrm{C}$ in peptone-yeast extract based medium (PY) [28]. Fructose, glucose, cellobiose $(30 \mathrm{mM}$; Sigma Chemical Co., St. Louis, MO, USA) or cellulose (1\% ball-milled Whatman No. 1 filter paper) was added to the medium as the sole energy source.

\subsection{Glucose Analogs}

Each organism was cultivated in PY containing glucose (PYG) and incubated at $39{ }^{\circ} \mathrm{C}$ for $16 \mathrm{~h}$. Aliquots $(0.1 \mathrm{~mL})$ of each culture were then anaerobically transferred via syringe [29] to tubes containing $10 \mathrm{~mL}$ of PYG or PYG plus $2 \mathrm{mM}$ of either methyl $\alpha$-D-glucopyranoside (methyl glucose; Sigma) or 2-deoxyglucose (Sigma). Inoculated tubes were then incubated at $39{ }^{\circ} \mathrm{C}$, and growth was monitored by measuring the increase of optical density with a spectrophotometer (Spectonic 20D, Milton Roy) at $650 \mathrm{~nm}$. This study was repeated twice and the results compiled.

A $2 \mathrm{mM}$ concentration of the glucose analog had no detectable inhibition upon normal growth of either organism in PYG (data not shown). Therefore, this concentration of analog exerted no toxic or inhibitory effect on glucose metabolism or overall cellular function. In addition, no growth was observed in inoculated PY when the analog was the sole energy source. So, any bacterial growth was the direct result of catabolism of cellulose or cellobiose, and not catabolism of the analog or components of the medium. Therefore, $2 \mathrm{mM}$ concentration of each analog was used in all subsequent experiments.

As an additional test, $2 \mathrm{mM}$ of each analog was added to medium and incubated at $39{ }^{\circ} \mathrm{C}$ for two weeks. These tubes were then inoculated with each organism and further incubated. The analogs were found to still have the same physiological effect on growth as freshly prepared analog. This verified that the analogs did not undergo a detectable degradation under incubation conditions. Thus, subsequent recovery of each organism was a physiological response, and not because the analog had degraded over time.

\subsection{Inhibition of Cellulolysis}

Each organism was initially cultivated for at least 12 generations in PY medium containing cellulose, cellobiose or glucose. Aliquots $(0.1 \mathrm{~mL})$ of each were 
then individually transferred into three bottles $(30 \mathrm{~mL})$ of medium containing cellulose and three bottles (30 $\mathrm{mL}$ ) of medium containing cellulose plus $2 \mathrm{mM}$ of glucose analog. Specifically, $2 \mathrm{mM}$ of methyl glucose was added to bottles containing C. cellulovorans, and $2 \mathrm{mM}$ of 2-deoxyglucose was added to bottles containing E. cellulosolvens. All bottles were then incubated with agitation $(100 \mathrm{rpm})$ at $39^{\circ} \mathrm{C}$.

Periodically, $1 \mathrm{~mL}$ of medium was anaerobically removed from each bottle, and serially diluted in anaerobically prepared dilution blanks $(0.1 \quad \mathrm{M}$ potassium phosphate, $\mathrm{pH}$ 7.0). Diluted samples were then inoculated into roll tubes [28] containing PYG. Roll tubes were incubated at $39{ }^{\circ} \mathrm{C}$ for $48 \mathrm{~h}( \pm 2 \mathrm{~h})$, after which colony forming units (CFU) were counted using standard counting procedures [28]. This study was repeated and the results compiled.

\subsection{Growth on Disaccharides}

Each organism was initially cultivated for a minimum of 12 generations in medium containing glucose, fructose or cellobiose. Aliquots $(0.1 \mathrm{~mL})$ from each of these cultures were then individually transferred into two tubes of PY $(10 \mathrm{~mL})$ containing cellobiose and two tubes of PY $(10 \mathrm{~mL})$ containing cellobiose and a glucose analog $(2 \mathrm{mM})$, as described above. Inoculated tubes were then incubated at $39^{\circ} \mathrm{C}$. Growth was monitored by spectrophotometrically measuring the increase of optical density, as described above. This study was repeated twice and the results compiled.

\section{Results and Discussion}

\subsection{Analog}

The utilization of soluble substrates has long been known to inhibit the simultaneous use of multiple carbon sources in a variety of bacteria $[2,30]$. Several previous studies have attempted to directly measure the effect of CCR on the cellulolysis of anaerobic cellulolytic bacteria. However, these studies have often been hindered by the presence of confounding factors [17]. These factors include a decline of medium $\mathrm{pH}$, nutrient competition between groups of organisms and inaccuracy of measuring substrate depletion from the medium. Since the experimental results from these studies have not been clear, it has remained uncertain if cellulolysis is directly inhibited by some form of CCR in some bacteria. Therefore, a more direct method of measuring repression was employed in this study using glucose analogs.

Since the analog does not serve as a catabolite for either organism, any bacterial growth was the direct result of cellulose or cellobiose catabolism and not utilization of the analog or other components of the medium. This approach enabled a direct measurement of cellulolysis and cellobiosis, making it unnecessary to determine the level of substrate depletion in the medium. Also, the use of analogs eliminated the confounding inhibitory effects of low $\mathrm{pH}$ on cellulolytic activity, since reduction of medium $\mathrm{pH}$ would only occur by the accumulation of metabolites from cellulose catabolism.

\subsection{Inhibition of Cellulolysis}

This study found that both organisms exhibited a slower rate of growth in the cellulose medium that contained the analog (Figs. 1 and 2). This indicated the analog reduced cellulolysis of these bacteria. Since the $2 \mathrm{mM}$ concentration of the analog did not inhibit growth of the organisms in medium containing glucose (data not shown), this reduction of growth indicates the analog exerted an inhibitory effect on the respective organisms' cellulolytic activity. This is consistent with a form of CCR stimulated by the analog.

Organisms initially cultivated in cellulose-containing medium were the least sensitive to any inhibitory effects of the analog (Figs. 1 and 2). In contrast, an inhibitory effect was most apparent when each organism was initially cultivated in glucose-containing medium. Both organisms recovered fully from the inhibition within $14 \mathrm{~d}$. 


\section{cellulovorans and Eubacterium cellulosolvens}

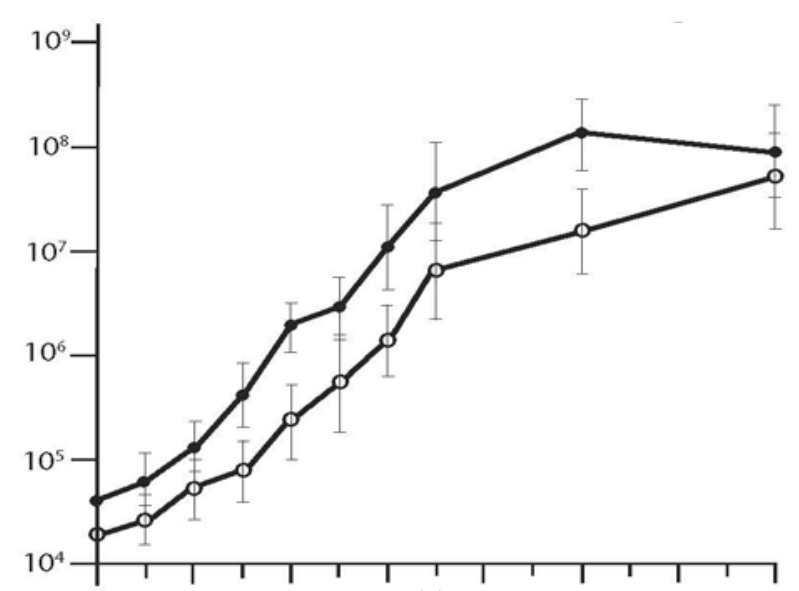

(a)

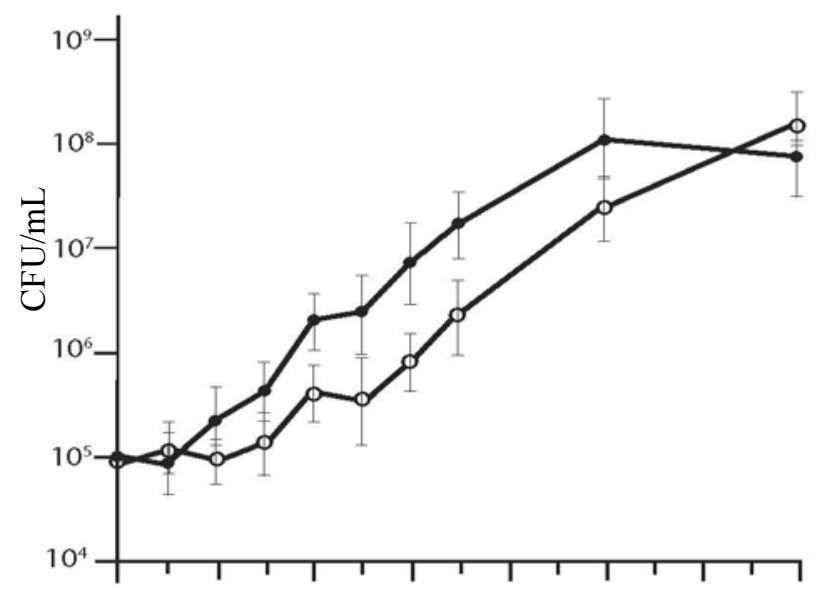

(b)

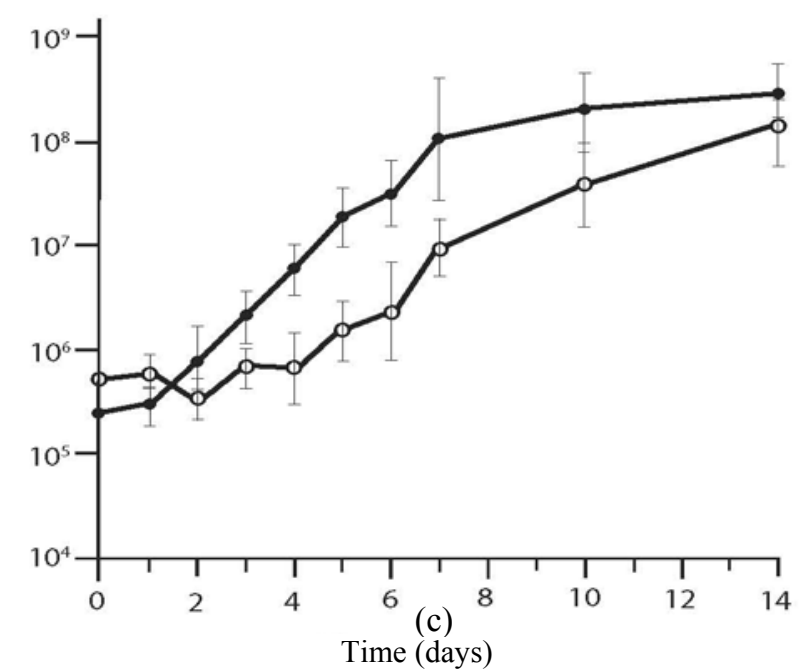

Fig. 1 Total bacterial count of cellulose-grown $C$. cellulovorans.

Cells were initially cultivated in medium containing cellulose (a), cellobiose (b) or glucose (c), and then subsequently cultivated in medium containing cellulose $(\bullet)$ or cellulose plus methylglucose $(\circ)$. Each data point represents the mean of two replicates with standard deviation bars shown.

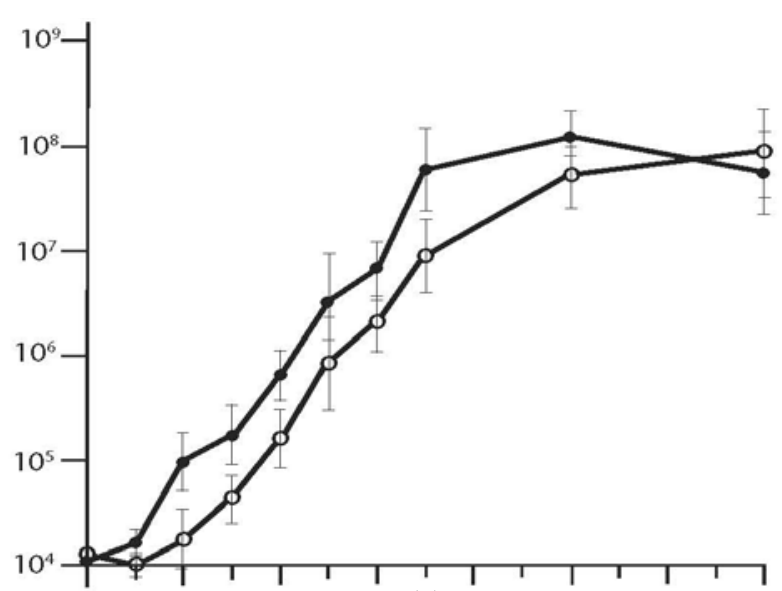

(a)

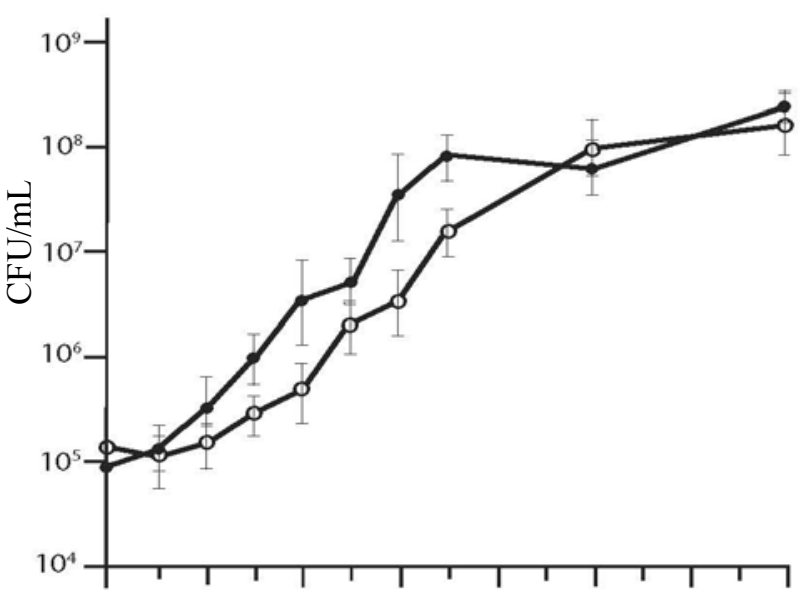

(b)

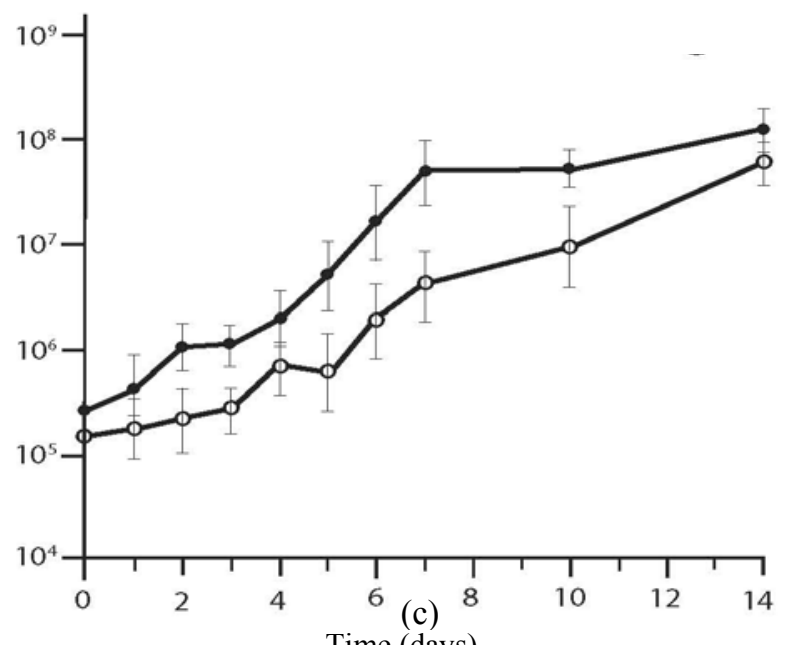

Fig. 2 Total bacterial count of cellulose-grown $E$. cellulosolvens.

Cells were initially cultivated in medium containing cellulose (a), cellobiose (b) or glucose (c), and then subsequently cultivated in medium containing cellulose $(\bullet)$ or cellulose plus 2-deoxyglucose $(\circ)$. Each data point represents the mean of two replicates with standard deviation bars shown. 
Previous work found that cellobiose induces portions of the cellulolytic system for the two organisms in this study [12, 13, 20, 22]. However, even when each organism was initially cultivated on cellobiose and transferred to cellulose-containing medium, we found the analog still inhibited growth (indicating CCR activity). Thus, initial cellobiose induction of some cellulolytic systems was still not sufficient to prevent cellulolysis from being inhibited by the analog. While growth in cellulose-medium was not completely inhibited during the $14 \mathrm{~d}$ incubation period, the level of inhibition detected was consistent with known CCR-mediated systems of repression for gram-positive bacteria [2, 6, 24]. Such repression will typically reduce the initial level of substrate utilization, but may not completely inhibit catabolism $[3,31,32]$.

Han et al. [33] report repression of some cellulolytic genes when mono-saccharides were added to cultures of $C$. cellulovorans in cellulose-containing medium. Following $10 \mathrm{~h}$ of incubation, expression of several cellulolytic genes began to increase. This is consistent with our results for both organisms. Inhibition was most severe immediately following introduction of the analog to the medium. Repression subsequently decreased during the remainder of the cultivation period.

Cellulose-grown E. cellulosolvens and $C$. cellulovorans have a cellulose-binding protein fraction that possesses cellulase activity $[12,13]$. This fraction is absent in cells cultivated in glucose-containing medium and is rapidly lost by cellulose-grown cells once glucose or a glucose analog is added to the medium $[12,13]$. This loss is consistent with a form of CCR acting on cellulolytic systems within these organisms, although the rapid loss of the protein fraction apparently involves a CCR mechanism not yet described or elucidated $[2,4,30]$. The analog's inhibition of cellulolysis, as observed in this study, is also consistent with the loss of these cellulose-inducible systems.

\subsection{Inhibition of Cellobiose Catabolism}

Measurement of the change in optical density also proved to be a sensitive and reliable method to measure inhibition of bacterial growth in cellobiose-containing medium. Interestingly, the respective analogs inhibited growth of both $E$. cellulosolvens and C. cellulovorans in cellobiose-containing medium, regardless of which energy substrate they were initially cultivated (Figs. 3 and 4). Thus, the analog repressed cellobiose catabolism even after the cell's cellobiose utilization system was fully induced. This is also consistent with our results described above, where initial cultivation on cellobiose did not fully relieve the analog inhibition of cellulolysis.

C. cellulovorans proved to be more sensitive to the effects of the analog than E. cellulosolven (Figs. 3 and 4). In addition, each organism was most sensitive to the analog when initially cultivated in fructose-containing medium (Figs. 3 and 4), and took the longest period of incubation to recover. All cultures in cellobiose plus analog-containing medium ultimately recovered from inhibition within $24 \mathrm{~h}$, except for C. cellulovorans initially cultivated in the fructose-containing medium (which failed to grow even after $24 \mathrm{~h}$ incubation).

Interestingly, the lowest level of whole-cell binding of cellulose was also detected in fructose-grown cells, where a binding level even below negative controls was detected for both organisms [12] (unpublished data of Anderson and Blair). Genes for some cellulosome components of C. cellulovorans are expressed in fructose-grown cells, but at a lower level than glucose-grown cells [33, 34]. Fructose-grown E. cellulosolvens possessed a distinctively different membrane fatty acid profile when compared to cellulose, glucose and cellobiose-grown cells [20]. We also found that transfer of fructose-grown cells into cellulose-containing media only sporadically resulted in growth of either bacterium, even when no analog was present (data not shown). This is consistent with 

cellulovorans and Eubacterium cellulosolvens

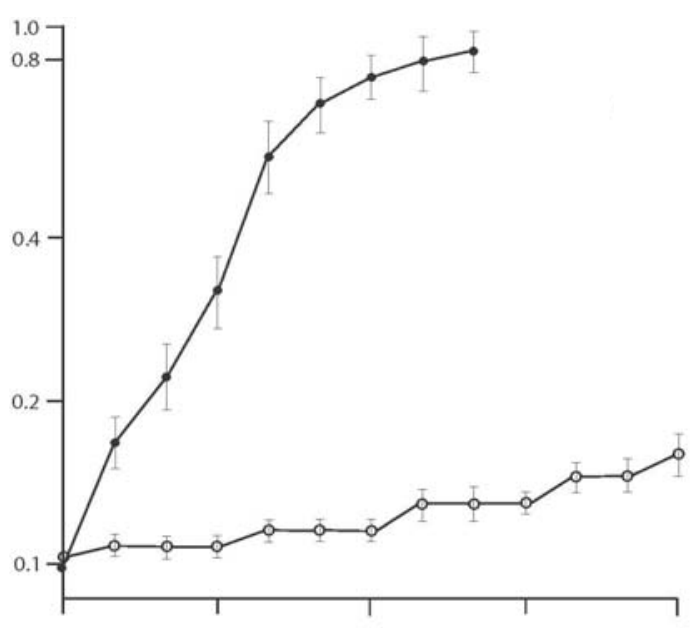

(a)

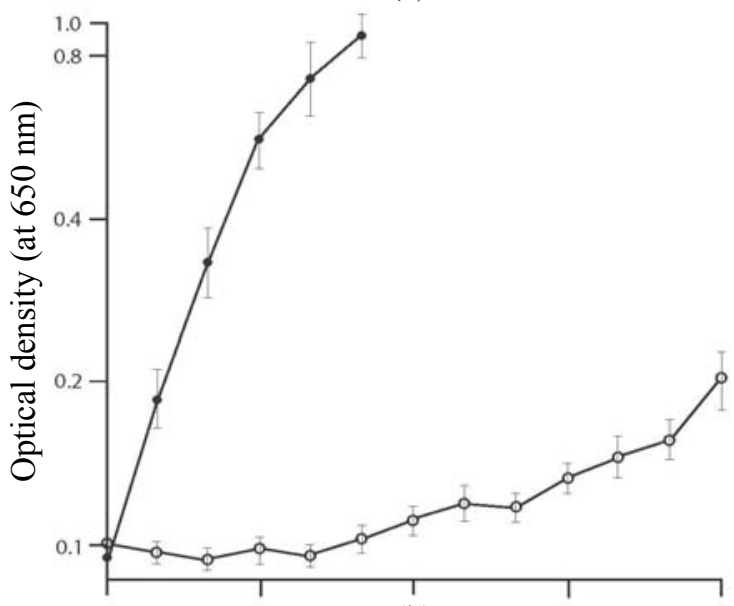

(b)

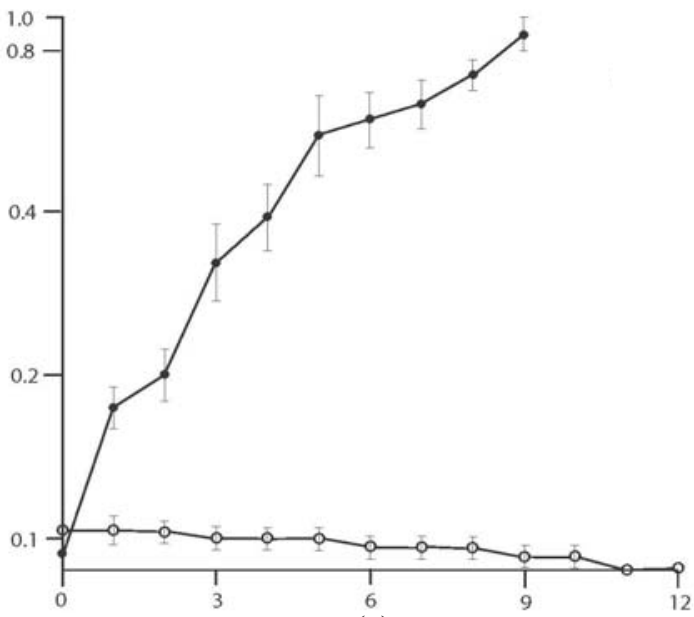

(c)

Hours

Fig. 3 Optical density of cellobiose-grown $C$. cellulovorans.

Cells were initially cultivated in medium containing glucose (a), cellobiose (b) or fructose (c), and then subsequently cultivated in medium containing cellobiose $(\bullet)$ or cellobiose plus methylglucose (०). Each data point represents the mean of four replicates with standard deviation bars shown.

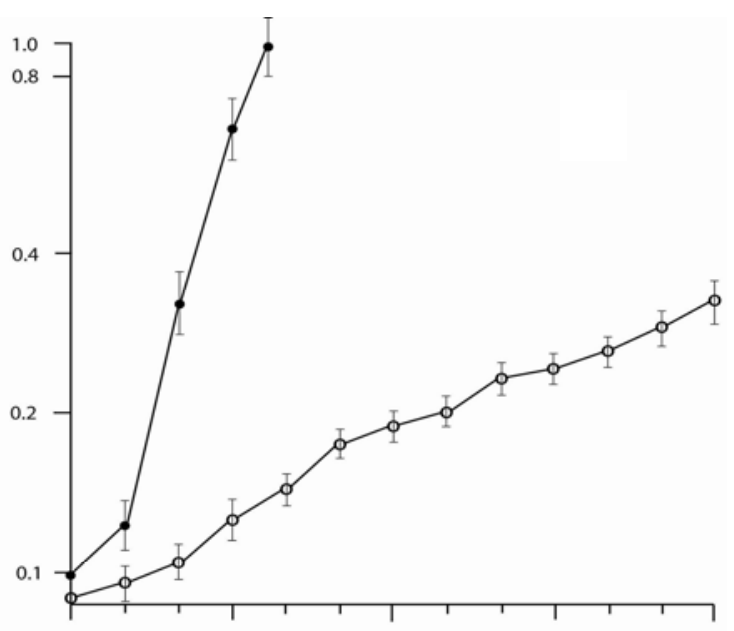

(a)
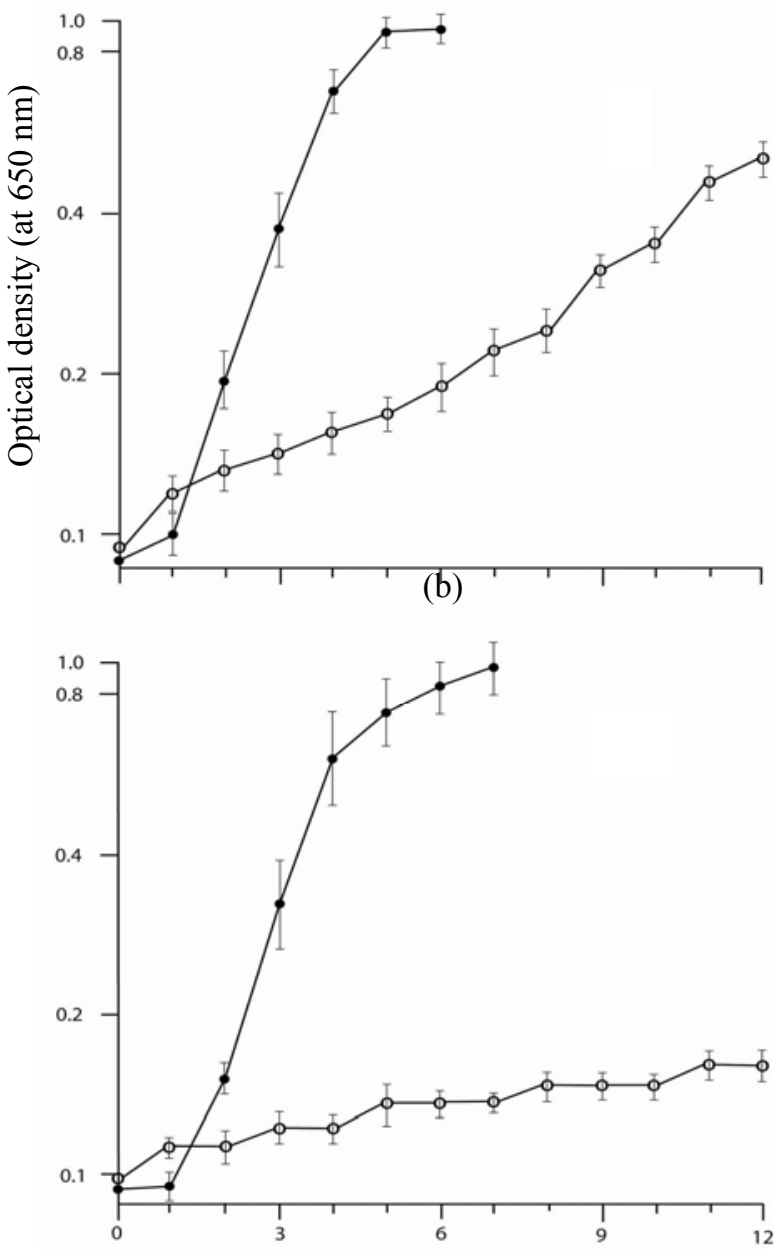

(c)

Hours

Fig. 4 Optical density of cellobiose-grown E. cellulosolvens. Cells were initially cultivated in medium containing glucose (a), cellobiose (b) or fructose (c), and then subsequently cultivated in medium containing cellobiose $(\bullet)$ or cellobiose plus 2-deoxyglucose (०). Each data point represents the mean of four replicates with standard deviation bars shown. 
our observation that fructose-grown cells are the most sensitive to repression.

A consequence of this observed CCR is that such repression may decrease the ability of these organisms to degrade cellulose in microbial environments where soluble carbohydrates are present. Such environments include both natural ecosystems (such as sediment or the gastrointestinal tract) and industrial biomass degradation processes. Thus, CCR could reduce these organisms' overall contribution to biomass degradation. However, many natural systems normally have a large consortium of microbes that work synergistically to reduce any free soluble carbohydrates. This consortium may force the cellulose degrading organisms to remain in a cellulolytic niche [35], and thereby preventing CCR from reducing overall bacterial cellulolysis. These consortiums may also neutralize other inhibitory factors (e.g., low $\mathrm{pH}$ and accumulation of metabolic inhibitors) that could reduce bacterial cellulolysis. This could demonstrate the importance of such consortiums (vs. pure cultures) in the recycling of biomass or production of biofuels [36].

\section{Conclusions}

The repression detected in both C. cellulovorans and E. cellulosolvens is consistent with a form of CCR. This repression may impact the cellulolytic activity of these organisms in a wide variety of ecological setting, both natural and industrial. However, the presence of other organisms that can reduce the surrounding levels of soluble carbohydrates, may enable C. cellulovorans and E. cellulosolvens to maintain active cellulolysis.

CCR could also represent an inhibitory variable in some natural and industrial cellulolytic processes that is not always identified or easily detected. This could be especially significant in pure culture fermentation platforms where no consortium is present to minimize the activity of CCR. Moreover, such CCR effects may not be readily accounted for in biomass conversion modeling or predicted during industrial "scale-up" processes. Thus, CCR could introduce an unexpected (and possibly unidentified) variable in these systems.

In addition, C. cellulolyticum has become a standard model for studying cellulolytic clostridia. However, our data revealed key differences in the regulatory systems of $C$. cellulovorans and $C$. cellulolyticum. For example, low levels of glucose apparently inhibit CCR in C. cellulolyticum, but we found low levels of glucose activated CCR in $C$. cellulovorans. This illustrates a significant regulatory differences among some of the cellulolytic clostridia, and highlights the difficulty of applying too broad of metabolic model to cellulolytic bacteria.

\section{Acknowledgments}

The authors thank Annette Olson for her technical assistance, and they dedicate this work to the memory of Penny Luna who provided much valuable laboratory assistance on this project.

\section{References}

[1] Magasanik, B. 1961. "Catabolite Repression." Cold Spring Harbor Symp. Quant. Biol. 26: 249-56.

[2] Deutscher, J., Francke, C., and Postma, P. W. 2006. "How Phosphotransferase System-Related Protein Phosphorylation Regulates Carbohydrate Metabolism in Bacteria.” Microbiol. Mol. Biol. Rev. 70 (4): 939-1031.

[3] Postma, P. W., Lengeler, J. W., and Jacobson, G. R. 1993. "Phosphoenolpyruvate: Carbohydrate Phosphotransferase Systems of Bacteria." Microbiol. Rev. 57 (3): 543-94.

[4] Deutscher, J. 2008. "The Mechanism of Carbon Catabolite Repression in Bacteria." Curr. Opin. Microbiol. 11 (2): 87-93.

[5] Vinuselvi, P., Kim, K., Sung, L., and Cheol-Min, G. 2012. "Rewiring Carbon Catabolite Repression for Microbial Cell Factory." BMB Re. 45 (2): 59-70.

[6] Singh, K. D., Schmalisch, M. H., Stülke, J., and Görke, B 2008. "Carbon Catabolite Repression in Bacillus subtilis: Quantitative Analysis of Repression Exerted by Different Carbon Sources.” J. Bacteriol. 190 (21): 7275-84.

[7] Wünsche, A., Hammer, E., Bartholomae, M., Völker, U., and Burkovski, A. 2012. "CcpA Forms Complexes with CodY and RpoA in Bacillus subtilis.” FEBS J. 279 (12): 2201-14.

[8] Meyer, F. M., Jules, M., Mehne, F. M. P., Coq, D. L., Landmann, J. J., Görke, B., Aymerich, S., and Stülke, J. 2011. "Malate-Mediated Carbon Catabolite Repression in 
Bacillus subtilis Involves the HPrK/CcpA Pathway." J. Bacteriol. 193 (24): 6939-46.

[9] Eppler, T., Postma, P., Schütz, A., Völker, U., and Boos, W. 2002. "Glycerol-3-Phosphate-Induced Catabolite Repression in Escherichia coli.” J. Bacteriol. 184 (11): 3044-52.

[10] Daniels, C., Godoy, P., Duque, E., Molina-Henares, M. A., de la Torre, J., del Arco, J. M., Herrera, C., Segura, A., Eugenia, M., Guazzaroni, M. E., Ferrer, M., and Ramos, J. L. 2010. "Global Regulation of Food Supply by Pseudomonas putida DOT-T1E.” J. Bacteriol. 192 (8): 2169-81.

[11] Bayer, E. A., and Lamed, R. 1986. "Ultrastructure of the Cell Surface Cellulosome of Clostridium thermocellulum and its Interaction with Cellulose." J. Bacteriol. 167 (3): 828-36.

[12] Blair, B. G., and Anderson, K. L. 1999. "Regulation of Cellulose-Inducible Structures of Clostridium cellulovorans." Can. J. Microbiol. 45 (3): 242-9.

[13] Blair, B. G., and Anderson, K. L. 1999. "Cellulose-Inducible Ultrastructural Protuberances and Cellulose-Affinity Proteins of Eubacterium cellulosolvens." Anaerobe 5 (5): 547-54.

[14] Abdou, L., Boileau, C., de Philip, P., Pagés, S., Fiérobe, H. P., and Tardif, C. 2008. "Transcriptional Regulation of the Clostridium cellulolyticum 'cip-cel' Operon: A Complex Mechanism Involving a Catabolite-Responsive Element.” J. Bacteriol. 190 (5): 1499-506.

[15] Doi, R., and Kosugi, A. 2004. "Cellulosomes: Plant-Cell-Wall-Degrading Enzyme Complexes." Nat. Rev. Microbiol. 2 (7): 541-51.

[16] Zhang, Yi-H. P., and Lynd, L. R. 2005. "Regulation of Cellulose Synthesis in Batch and Continuous Cultures of Clostridium thermocellum.” J. Bacteriol. 187 (1): 99-106.

[17] Anderson, K. L. 2000. "Degradation of Cellulose and Starch by Anaerobic Bacteria." In Glycomicrobiology. New York: Plenum Publishers, 359-86.

[18] Xu, C., Huang, R., Teng, L., Wang, D., Hemme, C., Borovok, I., He, Q., Lamed, R., Bayer, E. A., Zhou, J., and $\mathrm{Xu}, \mathrm{J}$. 2013. "Structure and Regulation of the Cellulose Degradome in Clostridium cellulyticum." Biotechnol. Biofuels 6: 73.

[19] Leschine, S. B. 1995. "Cellulose Degradation in Anaerobic Environments." Annual Rev. Microbiol. 49: 399-426.

[20] Moon, M., and Anderson, K. 2001. "Eubacterium cellulosolvens Alters Its Membrane Protein, Lipoprotein and Fatty Acid Composition in Response to Growth on Cellulose." Anaerobe 7 (4): 227-36.

[21] Yoda, K., Toyoda, A., Mukoyama, Y., Nakamura, Y., and Minato, H. 2005. "Cloning, Sequencing and Expression of a Eubacterium cellulosolvens 5 Gene
Encoding an Endoglucanase (Ce15A) with Novel Carbohydrate-Binding Modules and Properties of Cel5A.” Appl. Environ. Microbiol. 71 (10): 5787-93.

[22] Doi, R., and Tamaru, Y. 2001. "The Clostridum cellulovorans Cellulosome: An Enzyme Complex with Plant Cell Wall Degrading Activity." Chem. Rec. 1 (1): 24-32.

[23] Gancedo, J. M., and Gancedo, C. 1986. "Catabolite Repression Mutants in Yeast.” FEMS Microbiol. Rev. 32 (3-4): 179-87.

[24] Cvitkovitch, D. G., Boyd, D. A., and Hamilton, I. R. 1995. "Regulation of Sugar Transport via the Multiple Sugar Metabolism Operon of Streptococcus mutans by the Phosphoenolpyruvate Phosphotransferase System." J. Bacteriol. 177 (19): 5704-6.

[25] Saier, M. H., Novotny, M. J., Comeau-Fuhrman, D., Osumi, T., and Desai, J. D. 1983. "Cooperative Binding of the Sugar Substrates and Allosteric Regulatory Protein (Enzyme III ${ }^{\mathrm{Glc}}$ of the Phosphotransferase System) to Lactose and Melibiose Permeases in Escherichia coli and Salmonella typhimurium." J. Bacteriol. 155 (3): 1351-7.

[26] Prins, R. A., van Vugt, F., Hungate, R. E., and van Vorstenbosch, C. 1972. "A Comparison of Strains of Eubacterium cellulosolvens from the Rumen." Antonie van Leeuwen 38 (1): 153-61.

[27] Sleat, R., Mah, R. A., and Robinson, R. 1984. "Isolation and Characterization of an Anaerobic, Cellulolytic Bacterium, Clostridium cellulovorans sp. Nov." Appl. Environ. Microbiol. 48 (1): 88-93.

[28] Holdeman, L. V., Cato, E. P., and Moore, W. E. C. 1977. Anaerobe Laboratory Manual. Blacksburg, VA: Virginia Polytechnic Institute and State University, 37-9.

[29] Macy, J. M., Snellen, J. E., and Hungate, R. E. 1972. "Use of Syringe Methods for Anaerobiosis." Am. J. Clin. Nutr. 25 (12): 1318-23.

[30] Brückner, R., and Titgemeyer, F. 2002. "Carbon Catabolite Repression in Bacteria: Choice of Carbon Source and Autoregulatory Limitation of Sugar Utilization.” FEMS Microbiol. Lett. 209 (2): 141-8.

[31] Bassias, J., and Brückner, R. 1998. "Regulation of Lactose Utilization Genes in Staphylococcus xylosus." J. Bacteriol. 180 (9): 2273-9.

[32] Saier, M. H., Chauvaux, S., Cook, G. M., Deutscher, J., Paulsen, I. T., Reizer, J., and Ye, J. J. 1996. "Catabolite Repression and Inducer Control in Gram-Positive Bacteria." Microbiol. 142 (2): 217-30.

[33] Han, S. O., Yukawa, H., Inui, M., and Doi, R. H. 2003. "Regulation of Expression of Cellulosomal Cellulose and Hemicellulase Genes in Clostridium cellulovorans." J. Bacteriol. 185 (20): 6067-75.

[34] Matano, S., Park, J. S., Goldstein, M. A., and Doi, R. H. 
cellulovorans and Eubacterium cellulosolvens

1994. "Cellulose Promotes Extracellular Assembly of Clostridium cellulovorans Cellulosomes.” J. Bacteriol. 176 (22): 6952-6.

[35] Baldazzi, V., Ropers, D., Geiselmann, J., Kahn, D., and De Jong, H. 2012. "Importance of Metabolic Coupling for the Dynamics of Gene Expression Following Diauxic Shift in Escherichia coli." J. Theor. Biol. 295: 100-15.

[36] Zuroff, T. R., and Curis, W. R. 2012. "Developing Symbiotic Consortia for Lignocellulosic Biofuel Production.” Appl. Microbiol. Biotechnol. 93 (4): 1423-35. 